\title{
Malvengewächse der Nebelwüsten Chiles und Perus
}

\author{
Julio V. Schneider \& Marilú L. Huertas
}

\begin{abstract}
The fog oases of the coastal desert of Chile and Peru are an unique ecosystem in which the plants are maintained almost exclusively by fog humidity. One of the most prominent plant families of the area are the Malvaceae. They are well-adapted to the environmental conditions and a locally dominant element of the fog oases. Selected representatives are portrayed.
\end{abstract}

\section{Zusammenfassung}

Die Nebeloasen der chilenisch-peruanischen Küstenwüste sind ein einzigartiges Ökosystem, in dem die Pflanzen nahezu ausschließlich von der im Nebel enthaltenen Feuchtigkeit versorgt werden. Eine der artenreichsten und bestandsprägenden Pflanzenfamilien in diesem Ökosystem sind die Malvaceen, die in vielerlei Hinsicht an die dort herrschenden Umweltbedingungen angepasst sind und etliche attraktive Arten stellen. Ausgewählte Vertreter dieser Familie werden hier vorgestellt.

\section{Die Nebeloasen}

Die Atacama ist die bekannteste Wüste Südamerikas und eine der trockensten der Erde überhaupt. An einigen Orten ist seit Jahrzehnten kein einziger Tropfen Niederschlag mehr gefallen. Und auch über geologische Zeiträume hinweg betrachtet wartet die Atacama mit Superlativen auf: wie geologische Untersuchungen gezeigt haben, herrscht in diesem Gebiet seit 150 Millionen Jahren ununterbrochen ein halbtrockenes bis trockenes Klima (HartLEY et al. 2005). Es handelt sich somit um die älteste der heutigen Wüsten. Die auch in heutiger Zeit $\mathrm{zu}$ beobachtende extreme Trockenheit setzte vermutlich erstmals vor etwa 25 Millionen Jahren ein (Dunar et al. 2005). Als Ursachen gelten unter anderem die auftreibenden kalten Wassermassen des nordwärts entlang

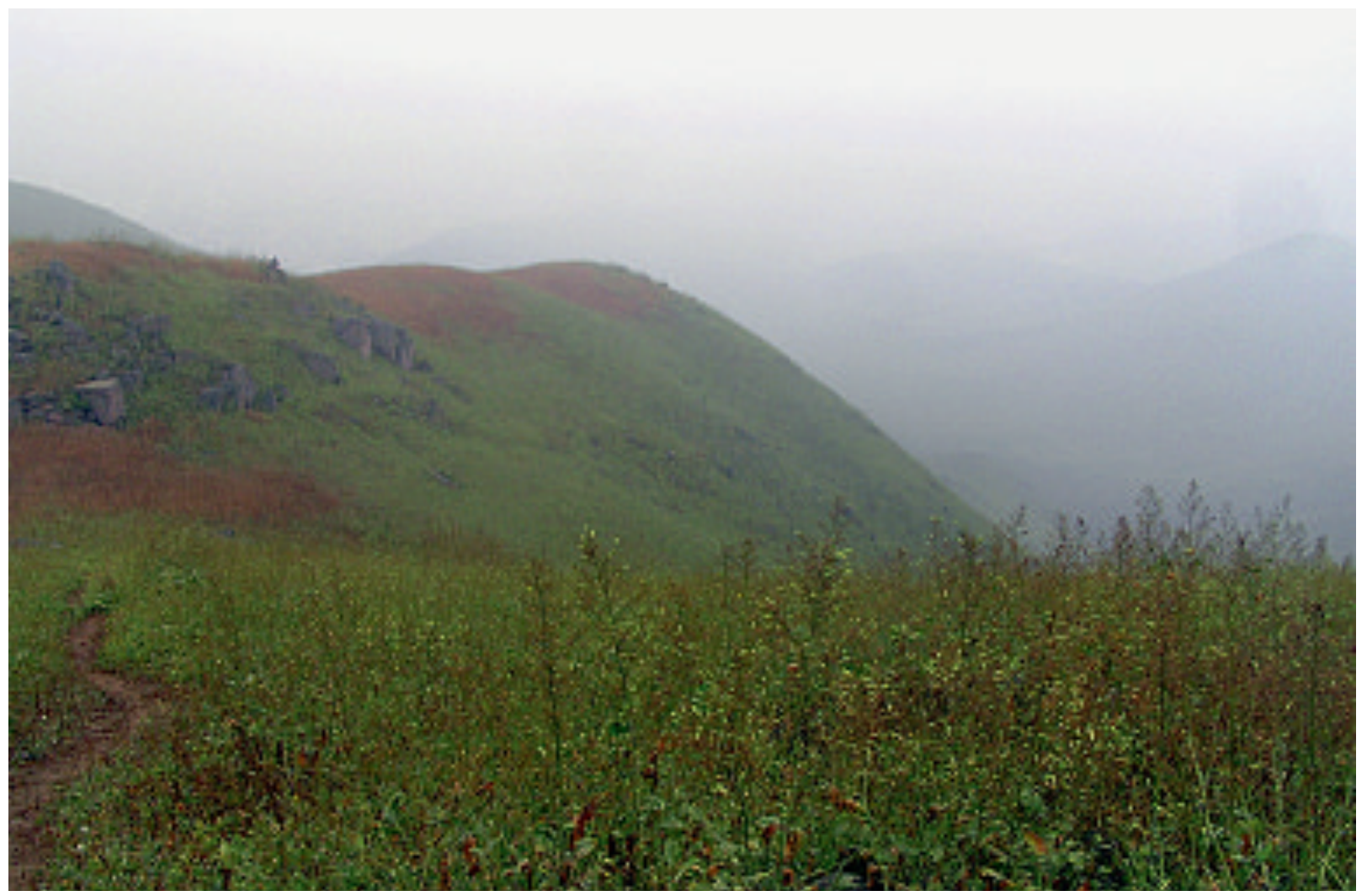



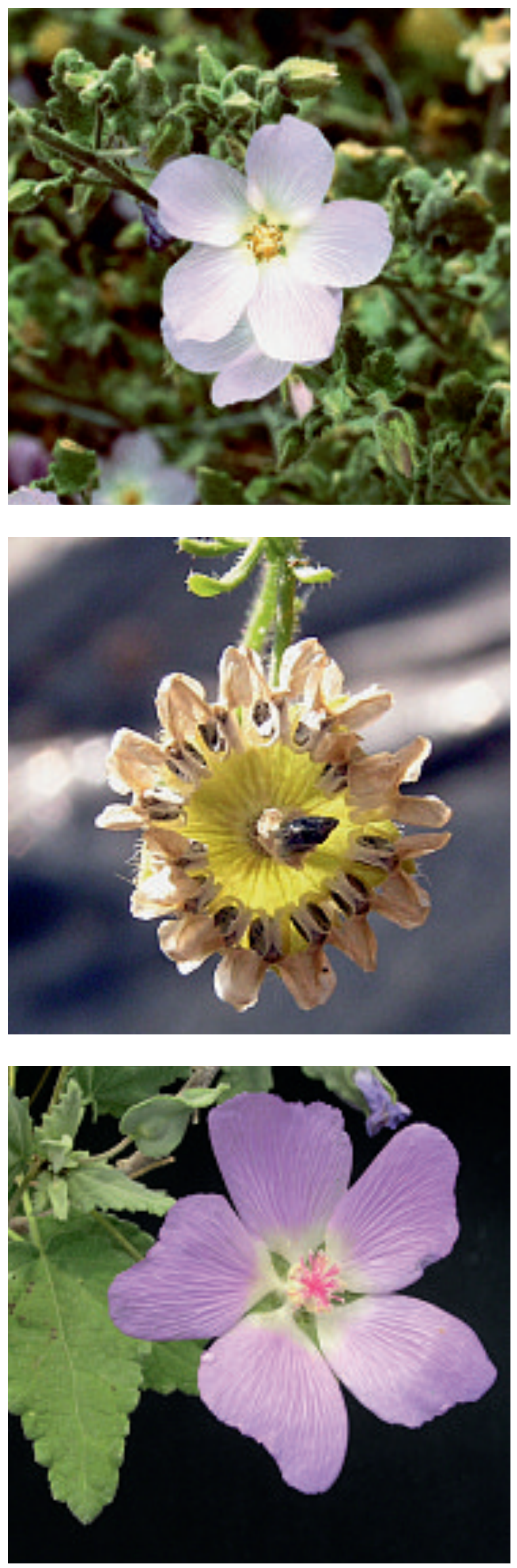

der Pazifikküste fließenden Humboldtstroms, durch den die Verdunstung herabgesetzt ist, und der Regenschatteneffekt, der nach der Hebung der Anden im Tertiär entstand. Diese Faktoren bewirken, dass messbare Niederschläge ein sehr seltenes Ereignis sind. Lediglich in den in unregelmäßigen Abständen wiederkehrenden El-Niño-Jahren finden nennenswerte Niederschläge statt, doch von diesen alleine könnten sich die Pflanzen der Wüste kaum am Leben halten.

Die Atacama-Wüste erstreckt sich über das nördliche Chile, und zwar von der Küstenebene bis in die Hochlagen der Andenkordillere. Allein daran lässt sich ermessen, dass die Atacama kein einheitlicher Lebensraum ist. Zwar ist allen Standorten eine extreme Trockenheit gemein, aber Unterschiede im Gelände, der Höhe und in der Entfernung von der Küste bewirken, dass sich unterschiedliche Vegetationstypen entwickeln konnten. Ein ganz besonderer sind die Lomas oder Nebeloasen (Abb. 1), wie sie auch genannt werden. Wie der Name schon vermuten lässt, spielt Nebelfeuchtigkeit eine entscheidende Rolle für das Überleben der Pflanzen. In den Wintermonaten der südlichen Hemisphäre bilden sich ausgedehnte Nebelbänke, die vom pazifischen Ozean auf die Küste zutreiben und große Mengen an Feuchtigkeit mitbringen. Die Nebel stauen sich an den in Küstennähe liegenden Hügeln und Bergen, die teils isoliert liegen, teils zusammenhängende Ketten bilden. An diesen bis ca. 1000 m hohen Stauzonen kulminiert nun die Nebelfeuchtig-

Abb. 1 (Seite 49): Lomas de Carabayllo, eine der Nebeloasen am Rande der peruanischen Hauptstadt Lima während der Vegetationsperiode; im Vordergrund eine Tabak-Art (Nicotiana paniculata).

Abb. 2 (Seite 50 oben): Cristaria integerrima, eine morphologisch sehr variable Art der nordchilenischen Nebeloasen in der Umgebung von Paposo.

Abb. 3 (Seite 50 Mitte): Karpocrater, die tellerförmige Basis des Säulchens, auf dem die Teilfrüchte bei Cristaria zur Fruchtreife stehen.

Abb. 4 (Seite 50 unten): Palaua trisepala, eine der großblütigen Arten aus Peru, die durch den Besitz von nur drei Kelchblättern gekennzeichnet ist. 
keit, und das machen sich die Pflanzen wiederum zunutze. Mit ihrem oberirdischen Vegetationskörper kämmen sie die Feuchtigkeit regelrecht aus. Untersuchungen haben gezeigt, dass ohne die Pflanzendecke die dem Boden zugeführte Feuchtigkeitsmenge deutlich niedriger ausfallen würde. Die Pflanzen sind somit ihre eigenen Gärtner. Man könnte meinen, die Nebelfeuchtigkeit würde nur einer eingeschränkten Anzahl an hoch spezialisierten Pflanzen das Überleben unter diesen vermeintlich unwirtlichen Bedingungen ermöglichen. Anpassungen an die Trockenheit weisen in der Tat viele Arten auf, so zum Beispiel die wasserspeichernden Organe der Kakteen, Mittagsblumen- oder Portulakgewächse. Aber entgegen der allgemeinen Erwartung findet sich in den Nebeloasen eine überaus reiche Flora mit etwa 1000 Blütenpflanzen-Arten (Dillon 1997a, b). Selbst Baumarten wie Carica candicans, eine Verwandte der Papaya, oder die zur Gerbstoffgewinnung verwendete, wirtschaftlich bedeutsame Tara (Caesalpinia spinosa), eine Vertreterin der Schmetterlingsblütler, haben in diesem Habitat ihre Nische gefunden. Der Artenreichtum ist aber nicht allein dem chilenischen Teil der AtacamaWüste und ihren Nebeloasen zuzuschreiben. Eher wenig bekannt ist, dass sich der küstennahe Wüstengürtel auch über einen Großteil der peruanischen Küste erstreckt, die für knapp die Hälfte der oben genannten Arten Heimat ist.

\section{Malvengewächse, erfolgreiche Strategen in den Nebeloasen}

Eines der auffälligsten Elemente der Flora der Nebeloasen sind die Malvengewächse (so nicht anders vermerkt, sind im Folgenden damit nur die Malvengewächse im engeren Sinne gemeint, d. h. die Vertreter der nach der aktuell aner-

Abb. 5 (oben): Palaua dissecta, eine weit verbreitete Art mit tief geteilten Blättern und leuchtenden Blüten.

Abb. 6 (Mitte): Palaua rhombifolia, in den Nebeloasen im Großraum der Hauptstadt Lima.

Abb. 7 (unten): Palaua moschata, die am weitesten verbreitete Art ihrer Gattung; hier vom Gipfel des Cerro Reque in Nordperu.
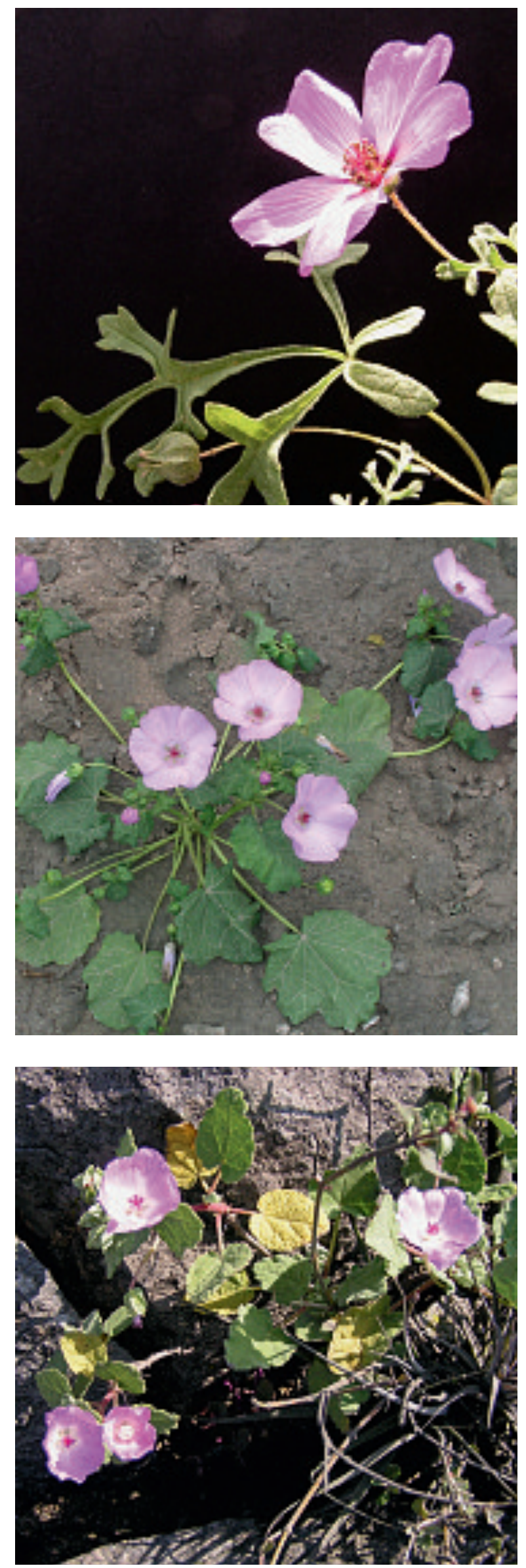

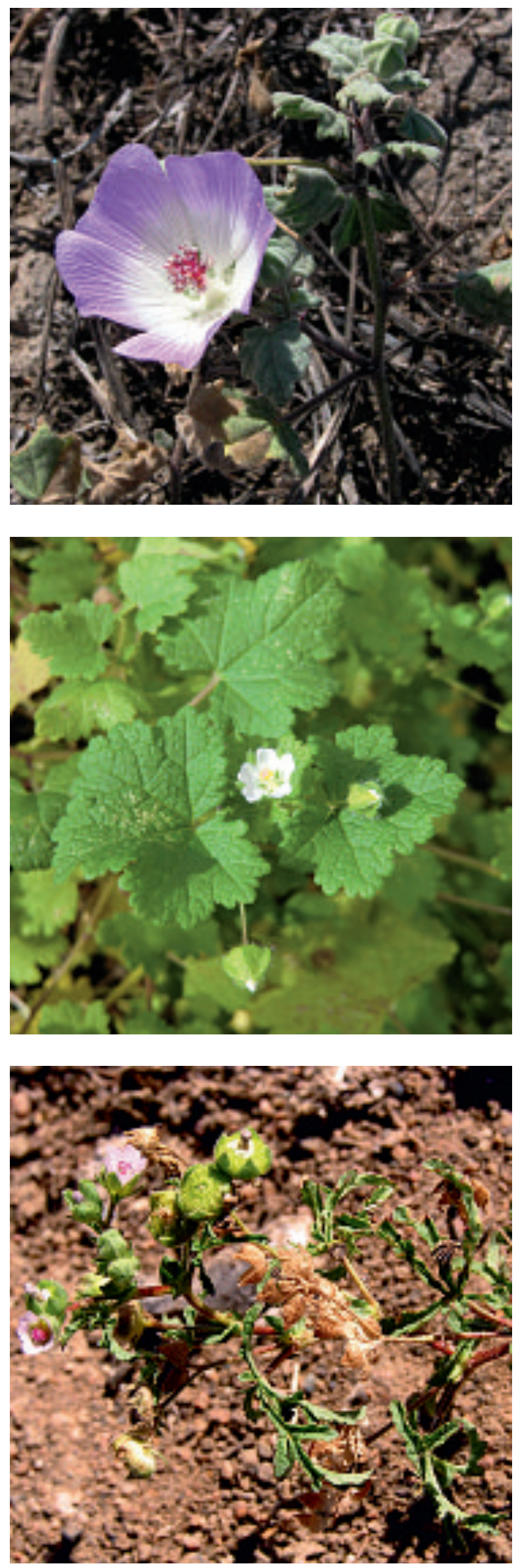

kannten Klassifikation nur als Unterfamilie der Malvaceae behandelten Malvoideen; siehe auch BAYER et al. 2010), nicht nur wegen ihrer zum Teil lebhaft gefärbten großen, attraktiven Blüten, sondern auch weil sie vielerorts in großen Individuenzahlen auftreten und somit zu den dominanten Arten zählen.

Dillon (2011) führt in seinen Florenlisten der Nebeloasen aktuell 19 Gattungen und ca. 74 Arten der Malvengewächse - nahezu ausschließlich Angehörige der Tribus Malveae auf, das sind also 7-8 Prozent der gesamten Flora. Selbst wenn aufgrund von Synonymisierung mancher Art noch einige Namen aus der Liste wegfallen, handelt es sich um eine beachtliche Vielfalt für ein derartiges Habitat. Das lässt darauf schließen, dass die Malvengewächse besonders erfolgreich an die in den Wüsten vorherrschenden Umweltbedingungen angepasst sind. Auf den ersten Blick erschließt sich das nicht, denn Wasserspeichergewebe sind nicht vorhanden. Doch die erfolgreiche Strategie liegt eher im Verborgenen. Viele Malvengewächse sind zum Beispiel außerordentlich flexibel hinsichtlich ihres Lebenszyklus, d. h. viele Arten sind in der Lage, in extrem trockenen Jahren ihren Lebenszyklus rasch abzuschließen und die ungünstige Jahreszeit in Form des Samens im Boden zu überdauern. Damit einher geht die Möglichkeit der Selbstbefruchtung, sollten die Bestäuber ausbleiben. Besonders wichtig erscheinen auch der gute Austrocknungsschutz und die enorme Langlebigkeit der Samen. Wie Untersuchungen an nordamerikanischen Malven zeigten, besitzen selbst über einhundert Jahre alte Samen noch eine hohe Keimungsrate (Telewski \& Zeevaart 2002). Aber auch im vegetativen Bereich finden sich Anpassungen

Abb. 8 (oben): Palaua tomentosa, ist eine morphologisch variable und nur in Südperu vorkommende Art, die mit Arten des Palaua-dissecta-Verwandtschaftskreises Hybriden bildet.

Abb. 9 (Mitte): Palaua modesta, eine seltene, besonders unscheinbare Art mit winzigen weißen Blüten, hier in der Nähe von Paposo (Nordchile) aufgenommen.

Abb. 10 (unten): Fuertesimalva chilensis in den Lomas de Atiquipa, Südperu. 
an die Trockenheit, so zum Beispiel ein dichtes Haarkleid an Spross und Blättern oder die Reduzierung der Blattoberfläche.

Die relativ große Artenvielfalt verdanken die Malvengewächse vor allem den beiden Gattungen Cristaria und Palaua, die etwa die Hälfte der Arten stellen. Beide Gattungen wurden in Rahmen eines am Forschungsinstitut und $\mathrm{Na}-$ turmuseum Senckenberg und der Universität Leipzig angesiedelten Forschungsprojekts zur Systematik und Evolution trockenheitsangepasster Malvengewächse untersucht.

Die Malvengewächse sind als Familie vergleichsweise einfach anzusprechen, da sie einen charakteristischen Blütenaufbau aufweisen: die fünfzählige Blütenhülle ist in Kelch und Krone gegliedert, unterhalb der Blüte ist oftmals ein Außenkelch vorhanden und die Staubblatt-Filamente entspringen der für die Familie charakteristischen Säule, in deren Zentrum die Griffel stehen. Die Früchte sind entweder Kapseln oder Spaltfrüchte, bei denen sich die Karpelle zur Fruchtreife in einzelne Teilfrüchte (Merikarpien) trennen.

Die mit etwa 21 Arten größte der in den Wüsten vorkommenden Gattungen der Malvengewächse ist Cristaria (Abb. 2; MuÑozScHick 1995). Sie hat ihren Verbreitungsschwerpunkt im chilenischen Teil der Wüste. Eine weit verbreitete Art, C. multifida, findet sich in Peru, mehrere Arten erreichen sogar Höhen von über $4000 \mathrm{~m}$ und drei Arten besiedeln die argentinische Seite der Anden, der Großteil ist aber auf die chilenischen Nebeloasen beschränkt. Neben kleinen einjährigen krautigen Pflanzen finden sich auch bis zu einem Meter hohe Sträucher. Die Blüten sind weiß, violett oder hellblau gefärbt, bei einigen Arten klein und unscheinbar, bei anderen aber bis zu $3 \mathrm{~cm}$ im Durchmesser. Als Anpassung an die extre-

Abb. 11 (oben): Fuertesimalva, peruviana eine Art, die meist mit $F$. chilensis vergesellschaftet ist und mit dieser vermutlich auch Hybriden bildet.

Abb. 12 (Mitte): Tarasa operculata, ein strauchförmiger Vertreter der Malvengewächse aus den Lomas de Carabayllo im Norden Limas.
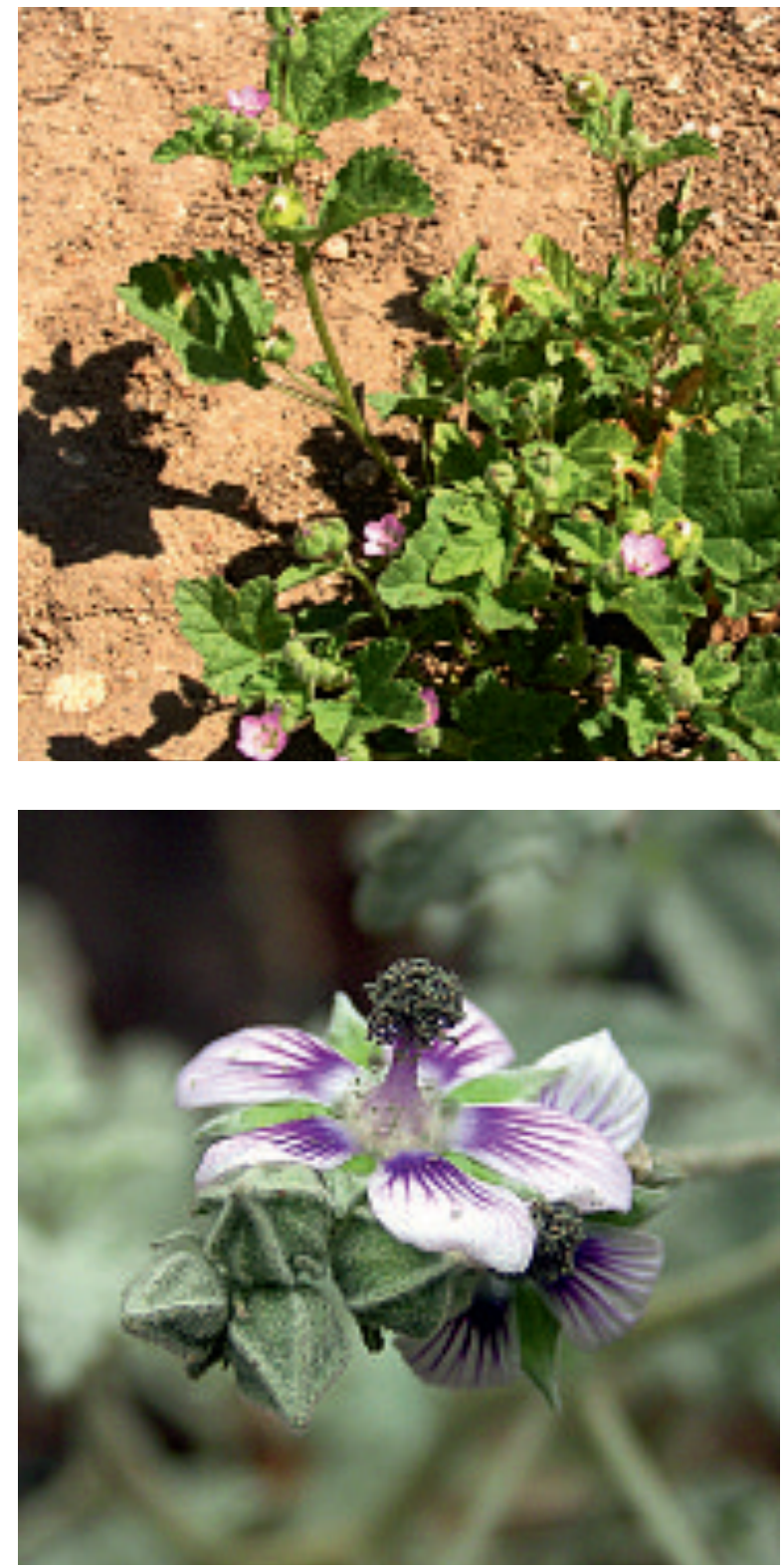

me Trockenheit sind die Blätter vieler Arten mit einem dichten Haarkleid ausgestattet oder aber in ihrer Oberfläche stark reduziert. Besonders auffällig sind die geflügelten Teilfrüchte, die im reifen Zustand eine Art Krone darstellen und der Ausbreitung der Samen dienen. Diesen seitwärts nach oben stehenden Flügeln, die eine Besonderheit innerhalb der Malvengewächse sind, hat die Gattung auch ihren Namen zu verdanken (lat. crista $=$ Kamm). In ähnlicher Weise ist dieses Merkmal nur in der nah verwandten Gattung Lecanophora zu beobachten. Einzigartig ist auch die stark verbreiterte Basis des zentralen Säulchens, durch die die Teilfrüchte wie auf einem Präsentierteller - im 


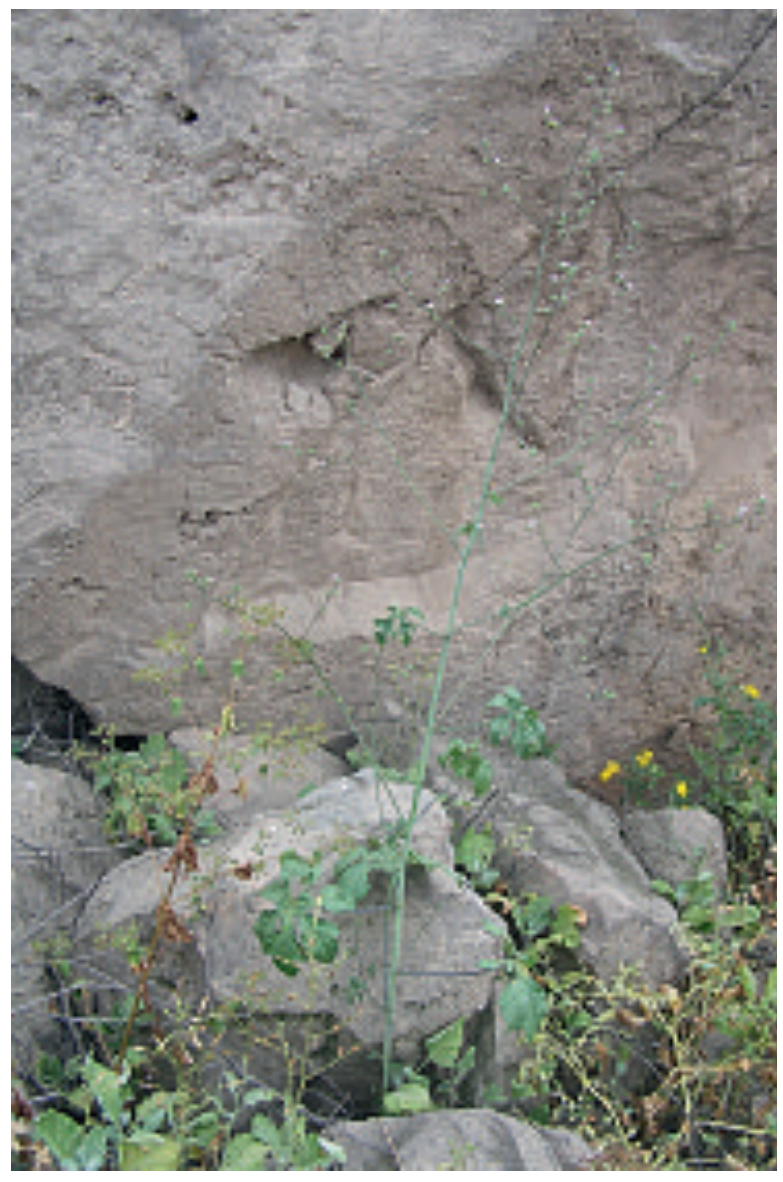

Fachjargon Karpocrater genannt - dargeboten werden (Abb. 3).

Wesentlich eingeschränkter in ihrem Verbreitungsgebiet ist die 15 Arten umfassende Gattung Palaua (Huertas et al. 2007, HuerTAs 2010). Ihr Areal sind nahezu ausschließlich die Nebeloasen, aber im Gegensatz zu Cristaria liegt ihr Schwerpunkt im peruanischen Teil der Wüste. Nur vier der Arten sind an wenigen Standorten in Chile zu finden. Auch Palaua weist ein sehr charakteristisches Merkmal im Fruchtbereich auf, das sie von allen anderen neuweltlichen Gattungen unterscheidet. Die Teilfrüchte sind nämlich nicht regelmäßig kreisförmig in einer Ebene angeordnet, sondern stehen unregelmäßig übereinander. Aufgrund dieses Merkmals wurde sie in früheren Klassifikationen zu den europäischen Gattungen Kitaibela und Malope gestellt, die einzigen Gattungen, die über einen vergleichbaren Aufbau der Früchte verfügen. Neuere Untersuchungen zeigten jedoch eindeutig, dass Palaua in einen anderen Verwandtschaftskreis zu stellen ist
(TATE et al. 2005). Interessant ist in diesem $\mathrm{Zu}$ sammenhang, dass eine Palaua-Art ( $P$. sandemanii) diesen charakteristischen Fruchtaufbau nicht aufweist. Sie wurde daher in der Vergangenheit zu verschiedenen Gattungen gestellt, bevor sie in Palaua ihre auch molekularsystematisch bestätigte Heimat fand. Bezüglich des Wuchsformenspektrums ist Palaua der Gattung Cristaria recht ähnlich: neben einjährigen kleinwüchsigen Arten gibt es solche mit verholzenden Sprossachsen. Auffallend sind die starken Unterschiede im Blüten- und Blütenstandsbereich innerhalb von Palaua. Die meisten Arten haben große violette Blüten, die einzeln in den Achseln laubblattartiger Tragblätter stehen (Abb. 4-8), einige Arten (P. inconspicua, P. modesta) besitzen aber nur wenige Millimeter große weiße Blüten (Abb. 9), die zudem in teils mehrblütigen Teilblütenständen stehen. Diese kleinen Blüten weisen auf ihre Tendenz zur Selbstbefruchtung hin, doch auch einige der großblütigen Arten haben sich als potenzielle Selbstbefruchter herausgestellt. Zwischen einigen der großblütigen Arten sind die Reproduktionsbarrieren unzulänglich ausgebildet, sodass Hybriden zu beobachten sind, wie kürzlich mit molekularen Methoden nachgewiesen werden konnte (SCHNEIDER et al. 2011).

Bezüglich ihrer Blüten weniger attraktiv, aber vielerorts bestandsbildend sind die Vertreter der Gattung Fuertesimalva, die durch den Besitz meist wickelförmiger Blütenstände auffällt. In den Nebeloasen weit verbreitet sind die beiden Arten Fuertesimalva chilensis (Abb. 10) und $F$. peruviana (Abb. 11). Beide sind echte Allrounder, denn ihr Verbreitungsgebiet beschränkt sich nicht nur auf die Nebeloasen, sondern erstreckt sich bis in die Hochlagen der Anden. So variabel ihre Verbreitung, so variabel auch ihre Morphologie. Zum einen spielen Umwelteinflüsse, zum anderen vermutlich Hybrid-

Abb. 13: Sida jatrophoides, eine der großwüchsigsten Vertreter der Malvaceen in den Nebelwüsten.

Abb. 14 (Seite 55): Die sehr seltene Gaya atiquipana bildet einen besonderen Farbtupfer vor dem erdfarbenen Hintergrund der Lomas. 


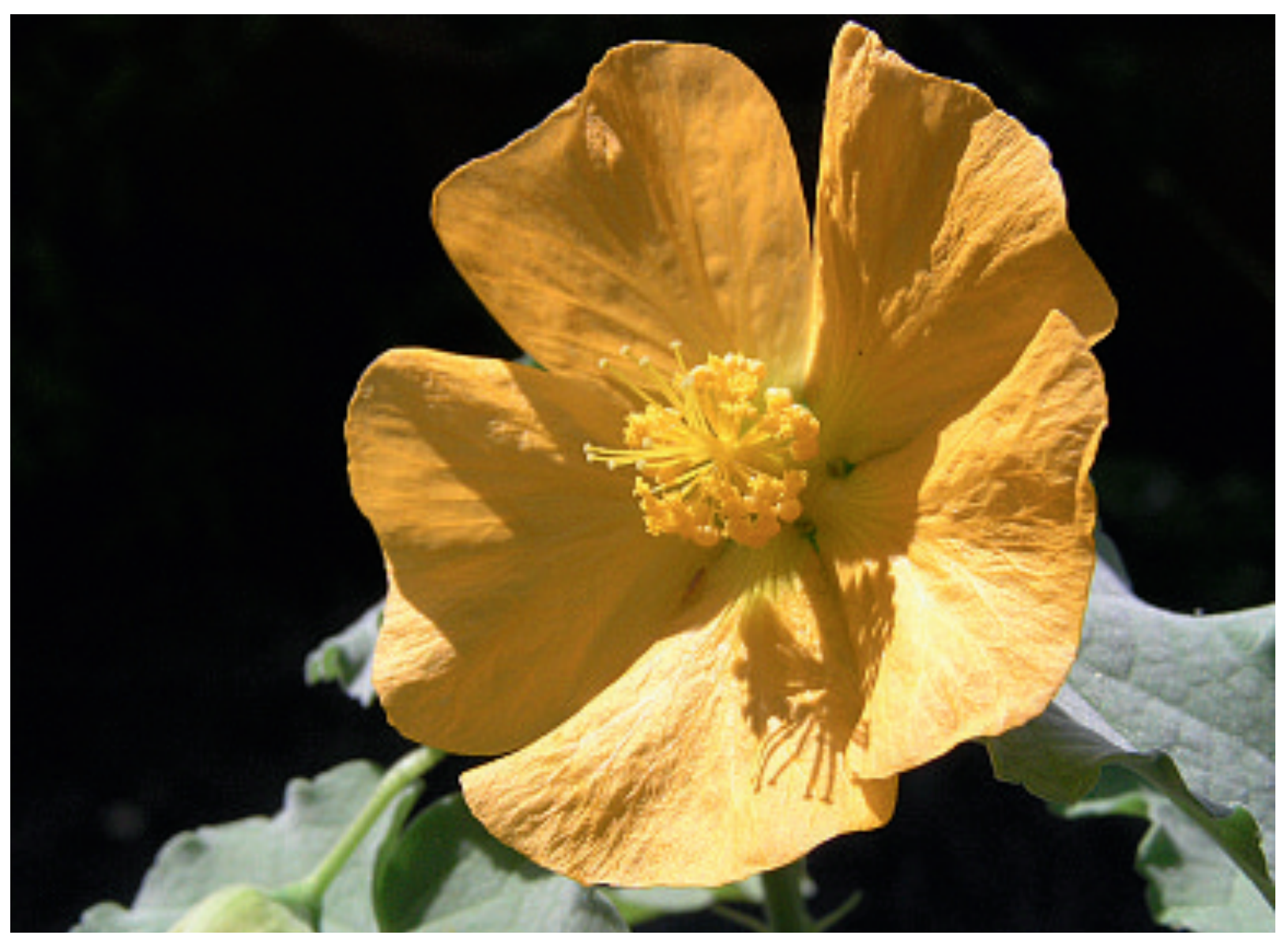

bildung eine wesentliche Rolle für die in vielen Fällen zu beobachtenden Übergänge in der Blatt- und Blütenmorphologie zwischen den Arten. Mit Fuertesimalva nah verwandt ist die Gattung Tarasa, die ebenfalls durch wickelförmige Blütenstände gekennzeichnet ist. Letztere ist allerdings ein eher seltener Vertreter in den Nebeloasen. Sie findet sich vielmehr in Höhen oberhalb von $3000 \mathrm{~m}$ und reicht bis in Zonen, in denen regelmäßig Nachtfröste herrschen. Interessant ist die Gattung vor allem hinsichtlich ihres ökologisch begründeten Dimorphismus. Die Frostzonen wurden von mehreren unabhängig voneinander entstandenen Arten besiedelt, jede davon aber durch einheitlich niedrigen Wuchs, Einjährigkeit und weiße bis hell-lavendelfarbene Blüten, deren Kronblätter nur bis zu $3 \mathrm{~mm}$ lang sind, gekennzeichnet (Tate \& Simpson 2003). Im Gegensatz dazu sind die Arten der tieferen Lagen meist strauchförmige, mehrjährige Arten und haben deutlich größere, kräftig lavendel- oder magentafarbene Blüten, so auch die Vertreter in den Nebeloasen wie Tarasa operculata (Abb. 12). Die mit über 100 Arten zu den artenreichsten Gattungen innerhalb der Malvaceen zählende Sida ist in den neu- und altweltlichen Tropen zu Hause, vereinzelt auch in den gemäßigten Zonen und kann ebenfalls einige wenige Arten in den $\mathrm{Ne}$ beloasen vorweisen, so zum Beispiel die dort weit verbreitete $S$. jatrophoides (Abb. 13). Es handelt sich um mehrjährige krautige oder strauchförmige Arten mit weißen, gelben, purpurnen, orange- oder rosafarbenen Blüten und Teilfrüchten, die oft durch zwei an der Spitze befindliche Zacken charakterisiert sind. Sida ist seit ihrer ursprünglichen Beschreibung durch Linné eine heterogene Gattung, weil er und seine Nachfolger dazu neigten, alle Arten, die keine Kapselfrüchte und keinen Außenkelch besaßen, ihr zuzuordnen (FrYXell 1997). Selbst in ihrer heutigen Umschreibung, d.h. nach der Ausgliederung zahlreicher Arten und deren Überführung in andere Gattungen, handelt es sich immer noch um eine nicht den natürlichen Verwandtschaftsverhältnissen ent- 


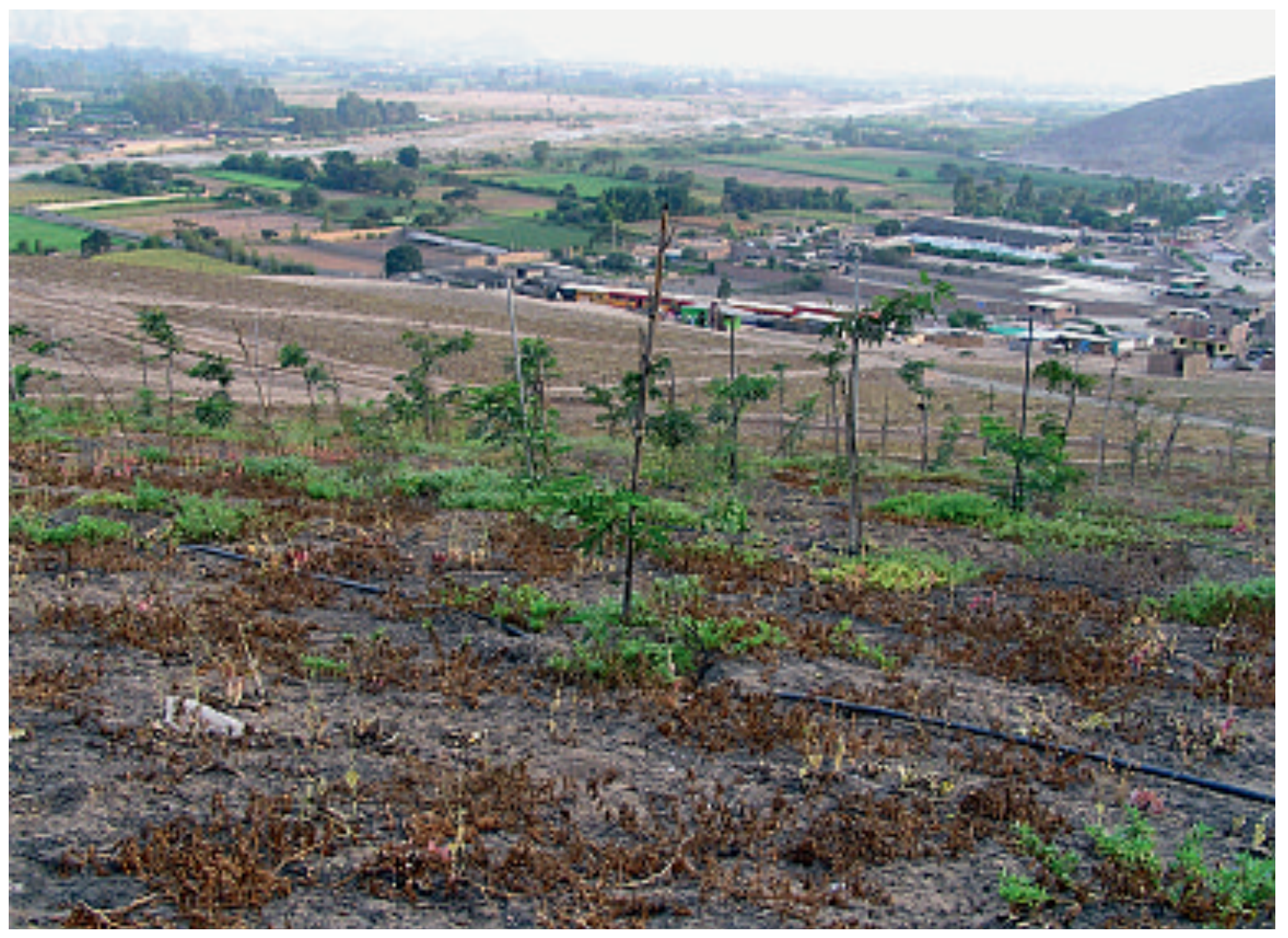

sprechende homogene Gruppe, wie molekulare Untersuchungen zeigten (Fuertes et al. 2003). Besonders prächtige Blüten lassen sich in der Gattung Gaya beobachten, deren Vertreter sich meist durch kräftig gelb gefärbte Kronen auszeichnen (Krapovickas 1995), wie die nur in Südperu vorkommende Gaya atiquipana (Abb. 14). Der Name Gaya leitet sich nicht von der griechischen Mythologie ab, sondern von dem französischen Botaniker JacQues ETIEnNe GaY (1786-1864), der viele Malven-Arten der Wüsten beschrieben hat. Wie in Cristaria, Palaua und Sida fehlt den Arten dieser Gattung ein Außenkelch. Auffällig sind zudem die meist leicht aufgeblasenen Früchte und ein zungenförmiger, den Samen umgebender Vorsprung im Fruchtinneren - das sogenannte Endoglossum -, der in dieser Gattung üblicherweise kammförmig ist.

Die hier in Kürze vorgestellten Gattungen gewähren einen Einblick in die Vielfalt der Malvengewächse in den Nebeloasen, eine Vielfalt, die allerdings trotz der scheinbar unwirtli- chen Umweltbedingungen vielerorts durch den Menschen in ihrem Bestand bedroht ist.

\section{Gefährdung der Malvengewächse in den Nebeloasen}

Trotz der extremen Trockenheit ist die chilenisch-peruanische Küstenwüste schon seit Jahrtausenden vom Menschen besiedelt. Insbesondere in Peru ist sie seit jeher Zentrum präkolumbischer Hochkulturen und nicht zuletzt Fundort der ältesten städtebaulichen Siedlung (Caral) des amerikanischen Kontinents. In neuerer Zeit sind vor allem die rasch wachsenden Städte Schuld daran, dass die Nebeloasen zurückgedrängt werden. Gerade in der Umgebung der peruanischen Hauptstadt Lima sind

Abb. 15: Sich in den Nebeloasen von Quebrada Verde in der Umgebung von Lima ausbreitende Siedlung mit landwirtschaftlich genutzten Flächen. Noch bis 2004 war dieser Standort unbesiedelt und beherbergte eine große Population der beiden Arten Palaua malvifolia und P. rhombifolia. 
viele Standorte der Nebeloasen bereits unwiederbringlich verlorengegangen (Abb. 15). Daher sind kleinräumig verbreitete Arten im Bereich der größeren Städte besonders gefährdet. Dies spiegelt sich auch in der Roten Liste der endemischen Pflanzen Perus wider (Chanco et al. 2006), in der etliche Arten als bedroht oder sogar stark bedroht eingestuft wurden, so z. B. Gaya atiquipana, Palaua camanensis und P. trisepala. Bisher gibt es mit den in Zentralperu gelegenen Lomas de Lachay leider nur ein Schutzgebiet im Bereich der Nebeloasen. Es bleibt daher zu hoffen, dass in naher Zukunft weitere Schutzgebiete folgen, um zumindest einige der gefährdeten Arten vor dem Aussterben zu bewahren.

\section{Dank}

Unser Dank gilt vor allem der Deutschen Forschungsgemeinschaft für die finanzielle Unterstützung im Rahmen des Schwerpunktprogramms SPP 1127 „Radiationen - Genese biologischer Diversität" sowie den peruanischen Kollegen César Cáceres, Asunción Cano, Magda Chanco, Leoncio Mariño, Amalia Rodríguez, José Roque und Abundio SagáSTEGUI, die uns während zahlreicher Feldaufenthalte hilfreich waren. Die Zweitautorin dankt außerdem der Friedrich-Ebert-Stiftung für ein Doktorandenstipendium.

\section{Literatur}

Bayer, C., Cole, T. C. H. \& Hilger, H. H. 2010: System der Blütenpflanzen - neue Zugehörigkeiten, neue Namen. -Palmengarten 74: 110-117.

Chanco, M., León, B. \& Sánchez, I. 2006: Malvaceae endémicas del Perú. - Rev. Peru. Biol. 13: 413s-425s. Dillon, M. O. 1997a: Lomas formations (Peru), CPD site SA42. In: Davis, S. D., Heywood, V. H., HerreraMacBryde, O., Villa-Lobos, J. \& Hamilton, A. (Hrsg.),
Centres of plant diversity: a guide and strategy for their conservation. Volume 3: The Americas. - Cambridge. Dillon, M. O. 1997b: Lomas formations of the Atacama desert (northern Chile), CPD site SA43. In: Davis, S. D., Heywood, V. H., Herrera-MacBryde, O., VillaLobos, J. \& Hamilton, A. (Hrsg.), Centres of plant diversity: a guide and strategy for their conservation. Volume 3: The Americas. - Cambridge.

Dillon, M. O. 2011: Flora of the lomas formations. http:// emuweb.fieldmuseum.org/botany/search_lomas.php.

Dunai, T. J., González López, G. A. \& Juez-Larré, J. 2005: Oligocene-Miocene age of aridity in the Atacama Desert revealed by exposure dating of erosion-sensitive landforms. - Geology 33: 321-324.

Fryxell, P. A. 1997: The American genera of Malvaceae - II. - Brittonia 49: 204-269.

Fuertes Aguilar, J. A., Fryxell, P. A. \& Jansen, R. K. 2003: Phylogenetic relationships and classification of the Sida generic alliance (Malvaceae) based on nrDNA ITS evidence. - Syst. Bot. 28: 352-364.

Hartley, A. J., Chong, G., Houston, J. \& Mather, A. E. 2005: 150 million years of climatic stability: evidence from the Atacama Desert, northern Chile. - J. Geol. Soc. 162: 421-424.

Huertas, M. L. 2010: Systematics and evolution of Palaua (Malvaceae). - Dissertation, Goethe-Universität, Frankfurt am Main.

Huertas, M. L., Schneider, J. V. \& Zizka, G. 2007:

Phylogenetic analysis of Palaua (Malveae, Malvaceae)

based on plastid and nuclear sequences. - Syst. Bot. 32: 157-165.

Krapovickas, A. 1996: Sinopsis del género Gaya

(Malvaceae). - Bonplandia 9: 57-87.

Muñoz-Schick, M. 1995. Revisión del género Cristaria (Malvaceae) en Chile. - Bol. Mus. Nac. Hist. Nat. 45:

45-110.

Schneider, J. V., Schulte, K., Fuertes Aguilar, J. \& Huertas, M. L. 2011: Molecular evidence for hybridization and introgression in the neotropical coastal desertendemic Palaua (Malveae, Malvaceae). - Mol. Phylogenet. Evol., 60: 373-384.

Tate, J. A. \& Simpson, B. B. 2003: Paraphyly of Tarasa (Malvaceae) and diverse origins of the polyploid species. - Syst. Bot. 28: 723-737.

Tate, J. A., Fuertes Aguilar, J., Wagstaff, S. J., La Duke, J.C., Slotta, T.A.B. \& Simpson, B. B. 2005: Phylogenetic relationships within the tribe Malveae (Malvaceae, subfamily Malvoideae) as inferred from ITS sequence data. - Amer. J. Bot. 92: 584-602.

Telewski, F. W. \& Zeevaart, J. A. D. 2002: The 120-yr period for Dr. BEAL's seed viability experiment. - Amer. J. Bot. 89: 1285-1288. 


\title{
Elsbeere - Baum des Jahres 2011
}

\author{
Veit Martin Dörken
}

\begin{abstract}
Wild service tree (Sorbus torminalis) is tree of the year 2011. Its biology, ecology and use are summarized.
\end{abstract}

\section{Zusammenfassung}

Die Elsbeere (Sorbus torminalis) ist Baum des Jahres 2011. Ihre Biologie, Ökologie und ihr Nutzen werden vorgestellt.

\section{Einleitung}

Die Elsbeere (Sorbus torminalis) wurde 2011 zum 21. Baum des Jahres gekürt. Dies ist neben dem seltenen Speierling (Sorbus domestica, Jahresbaum 1993) und der für Vögel wichtigen Futterpflanze Eberesche (Sorbus aucuparia, Jahresbaum 1997) nun bereits die dritte Art aus der Gattung Sorbus. Wichtige Auswahlkriterien für die Ernennung sind z. B. Seltenheit und Bedrohung. Es gibt aber auch ästhetische, ökologische und landschaftsgestalterische Aspekte. Sorbus torminalis ist zweifelsohne eine Baum-

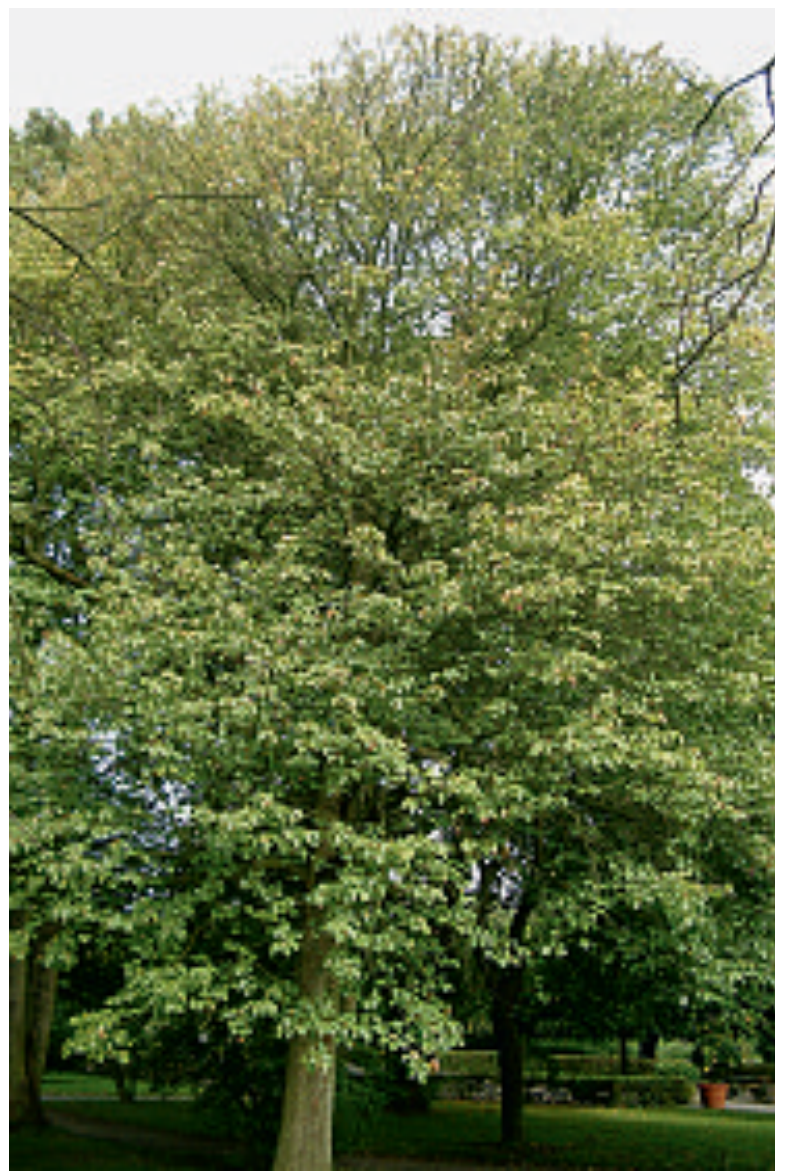

art, die der breiten Öffentlichkeit heute weitgehend unbekannt ist, obwohl sie früher als Wildobstgehölz geschätzt war. Dies war entscheidender Grund für die Ernennung zum Baum des Jahres. Weitere Auswahlkriterien waren ihre Seltenheit, das wertvolle harte Holz, die spektakuläre Herbstfärbung und ihr relativ später Blütezeitpunkt (Roloff 2011).

\section{Systematik}

Die Elsbeere gehört zur großen und vielgestaltigen Familie der Rosengewächse (Rosaceae). Sie wird zusammen mit den ebenfalls einheimischen Gattungen Amelanchier (Felsenbirne), Malus (Apfel) und Pyrus (Birne) zur Unterfamilie der Maloideae (= Apfelartigen) gestellt. Auch die Gattung Sorbus selbst ist artenreich (je nach Auffassung rund 140 Arten) und vielgestaltig. In Deutschland kommen nach der Auflistung bei Buttlen \& Hand (2008) 41 heimische Sorbus-Arten vor, von denen 22 erst 2005 neu beschrieben wurden (Meyer et al. 2005). Es gibt verschiedene Ansichten zur Gliederung der Gattung. Im einfachsten Fall gibt es nur zwei Untergattungen: Aucuparia (Ebereschen mit gefiedertem Laub) und Aria (Mehlbeeren mit ungefiedertem Laub). Nach dieser Einteilung gehört S. torminalis zur Untergattung Aria (Kelly \& Hillier 2004). Andere systematische Gliederungen sind dagegen komplizierter. So unterteilt z. B. Mabberley (2008) die Gattung in vier Untergattungen, nämlich in Aria, Cormus, Torminaria und Sorbus. Meyer et al. (2005) fügen diesen noch eine fünfte Untergat-

Abb. 1: Habitus einer Elsbeere. 